\title{
MAXIMAL USING OF SPECIFICS OF SOME BOUNDARY PROBLEMS IN POTENTIAL THEORY AFTER THEIR NUMERICAL ANALYSIS
}

\author{
L.I. Mochurad ${ }^{1)}$, Y.S. Harasym ${ }^{2)}$, B.A. Ostudin ${ }^{3)}$ \\ 1) Post-Graduate Student of the Department of Computational Mathematics, Ivan Franko National University in Lviv, \\ Mochurad_lesya@ukr.net, http://blues.lnu.edu.ua/ami/kom/mochurad \\ ${ }^{2)}$ Senior Lecturer of the Department of Computational Mathematics, Ivan Franko National University in Lviv, \\ garasym@yahoo.com, http://blues.lnu.edu.ua/ami/kom/garasym \\ 3) Associate Professor of the Department of Computational Mathematics, Ivan Franko National University in Lviv, \\ kom@franko.lviv.ua, http://blues.lnu.edu.ua/ami/kom/ostudin
}

Summary: some typical problems in the numerical analysis of certain types of boundary value problems of the potential theory in substantially spatial formulation are considered. On the basis of the integral equation method (IE) an approximate scheme of solving one model example is built and investigated. It is also considered that the doubly connected open surface where boundary conditions are set obtains the Abelian group of symmetry of the eighth order. This article shows how using the apparatus of the group theory it is possible to solve an initial problem by the help of the sequence of the eight independent IEs, where the integration is realized only on one of the congruent constituents of the surface. It creates the conditions for two parallel processes of problem solution in general. The collocation method for obtaining approximate values of needed "density of charge distribution" in the particular two-dimensional integral equations is used. To take into account the singular way of solving the problem in the circuit of the open surface the a posteriori method of error evaluation is created and the procedure of integrating clarification of solving the task in the mesh node is implemented. To prove the reliability and estimation of the technique efficiency the number of numerical experiments is carried out including the use of so called "plane" approximation of the examined spatial problem.

Keywords: the potential theory, integral equations, the collocation method, the Abelian group of symmetry, matrix of Fourier transformation, the a posteriori error evaluation, integrating clarification of solving.

\section{INTRODUCTION}

In the process of solving boundary value problems of the potential theory in electron optics the problem occurs when measuring the electrostatic field which is formed by the combination of a great number of the charged electrodes of complicated configuration. Thus, traditional application of IE method becomes substantially complicated as it is related to unknown quantities estimation mainly on the boundary surfaces. Therefore, the application of the group theory apparatus turns out to be more effective for the types of boundary tasks which obtain the Abelian group of symmetry of the finite order. However, taking into account this peculiarity in solving particular problems requires an individual approach. Although there are considerable advantages which allow to find approximate solutions with high accuracy.

\section{PROBLEM STATING}

Without loss of generality, we will consider the task of calculation of the electrostatic field of parallel condenser which is shown on the Pic. 1.

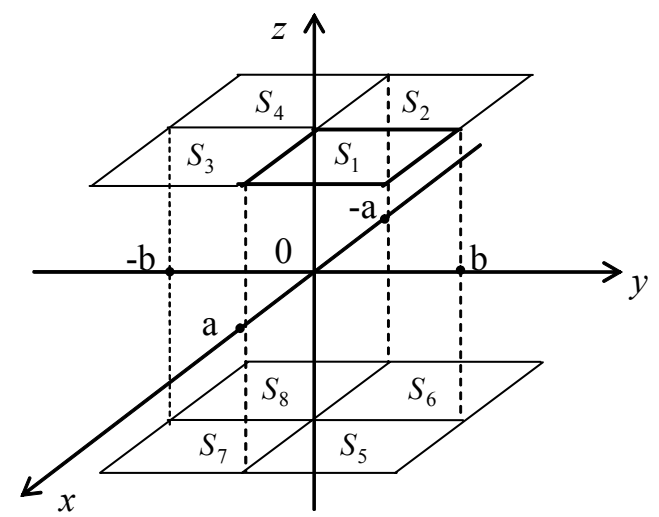

Pic. 1 - Position of parallel condenser disks

It is easy to see that in the mathematical modeling the information on the geometry of the charged electrodes is shown as some aggregate $S$ of two parallel rectangular disks. These will be considered as infinitely thin disks, with two-sides 
and limited piecewise smooth circuits of the finite length. From the mathematical point of view it is necessary to find a function $u(P) \in H^{1}\left(\Omega_{s}^{-}, \Delta\right)$, which satisfies the following conditions

$$
\begin{gathered}
\Delta u=0 \quad \text { в } \Omega_{s}^{-}:=\mathbf{R}^{3} \backslash \bar{S} ; \\
\delta^{ \pm} u=g_{ \pm} \text {on } S ; \\
\lim _{|P| \rightarrow \infty} u(P)=0, \quad P \in \Omega_{s}^{-},
\end{gathered}
$$

where $\delta^{ \pm}: H^{1}\left(\Omega_{s}^{-}\right) \rightarrow H^{1 / 2}(S)$ - the operators of track [1], $g_{ \pm} \in H^{1 / 2}(S)$ - set functions, where $g_{ \pm}-$known value of the searching function on $S$ from a positive and negative side accordingly, and

$$
H^{1}\left(\Omega_{s}^{-}, \Delta\right):=\left\{u \mid u \in H^{1}\left(\Omega_{s}^{-}\right), \Delta u \in L_{2}\left(\Omega_{s}^{-}\right)\right\} .
$$

During the electrostatic interpretation (1)-(3) $g_{+}(P)=g_{-}(P)=f(P)(P \in S)$ - the boundary potential value, which on each of two electrodes takes a permanent value. It is necessary to notice that these values in general do not possess symmetry or anti symmetry.

It is known [2] that the problem (1)-(3) is equivalent to such IE as

$$
\iint_{S} K(P, M) \rho(M) \mathrm{d} S_{M}=f(P), \quad P \in S,(4)
$$

where $K(P, M):=1 / \operatorname{dist}(P, M)$ - a fundamental solution to Laplace's equation in $\mathbf{R}^{3}$, and $\rho(M)$ is the needed "density of charge distribution" on $S$.

\section{ACCOUNTING PRESENT SYMMETRY}

The surface $S$ can be viewed as an aggregate of eight congruent constituents $S_{i}(i=\overline{1,8})$ (see Pic. 1). We will give (4) in accordance with a such partitioning of $S$ as an IE system

$$
\begin{aligned}
& \left\{\begin{array}{l}
\sum_{i=1}^{4} \iint_{\Delta_{i}} \rho_{i}(x, y) K\left(x, y ; x_{0}, y_{0}\right) \mathrm{d} x \mathrm{~d} y+ \\
+\sum_{i=5}^{8} \iint_{\Delta_{i}} \rho_{i}(x, y) K_{h}\left(x, y ; x_{0}, y_{0}\right) \mathrm{d} x \mathrm{~d} y= \\
=f_{k}\left(x_{0}, y_{0}\right), \quad\left(x_{0}, y_{0}\right) \in S_{k}, k=\overline{1,4} ; \\
\sum_{i=1}^{4} \iint_{\Delta_{i}} \rho_{i}(x, y) K_{h}\left(x, y ; x_{0}, y_{0}\right) \mathrm{d} x \mathrm{~d} y+ \\
+\sum_{i=5}^{8} \iint_{\Delta_{i}} \rho_{i}(x, y) K\left(x, y ; x_{0}, y_{0}\right) \mathrm{d} x \mathrm{~d} y= \\
=f_{k}\left(x_{0}, y_{0}\right), \quad\left(x_{0}, y_{0}\right) \in S_{k}, k=\overline{5,8} .
\end{array}\right. \\
& \text { Here } \quad\left\{\rho_{i}(x, y),(x, y) \in \Delta_{i}, i=\overline{1,8}\right\} \quad \text { is } \quad \text { a }
\end{aligned}
$$

generalized "density of charge distribution" on $S$;

$$
\begin{aligned}
\Delta_{1}=\Delta_{5} & :=[0, a] \times[0, b], \\
\Delta_{2}=\Delta_{6} & :=[-a, 0] \times[0, b], \\
\Delta_{3}=\Delta_{7} & :=[0, a] \times[-b, 0], \\
\Delta_{4}=\Delta_{8} & :=[-a, 0] \times[-b, 0] ; \\
K\left(x, y ; x_{0}, y_{0}\right) & :=\frac{1}{\sqrt{\left(x-x_{0}\right)^{2}+\left(y-y_{0}\right)^{2}}} ; \\
K_{h}\left(x, y ; x_{0}, y_{0}\right) & :=\frac{1}{\sqrt{\left(x-x_{0}\right)^{2}+\left(y-y_{0}\right)^{2}+4 h^{2}}} ;
\end{aligned}
$$

$2 h$ - the distance between disks.

Further, we will take into account that the surface $S$ obtains the Abelian group of symmetry of the eighth order $\left\{\tau_{i}\right\}_{i=1}^{8}$, which, in its turn, is a direct product of Abelian sub-groups $\left\{e, \tau_{x}\right\},\left\{e, \tau_{y}\right\}$, $\left\{e, \tau_{z}\right\}$, where $e-$ identical transformation, and $\tau_{x}, \tau_{y}, \tau_{z}-$ mirror reflection from three pairwise orthogonal planes $\{y z\},\{x z\},\{x y\}$, accordingly. So that the elements of the group are the following linear transformations: $\tau_{1}:=e, \tau_{2}:=\tau_{x}, \tau_{3}:=\tau_{y}$, $\tau_{4}:=\tau_{x} \circ \tau_{y}, \tau_{5}:=\tau_{z}, \tau_{6}:=\tau_{x} \circ \tau_{z}, \tau_{7}:=\tau_{y} \circ \tau_{z}$, $\tau_{8}:=\tau_{x} \circ \tau_{y} \circ \tau_{z}$. As unknown functions $\rho_{i}(x, y)$ depend only on two independent arguments, it is obvious that

$$
\begin{gathered}
\tau_{1}=\tau_{5}=\tau_{1}^{-1}=\tau_{5}^{-1}=\left(\begin{array}{ll}
1 & 0 \\
0 & 1
\end{array}\right), \\
\tau_{2}=\tau_{6}=\tau_{2}^{-1}=\tau_{6}^{-1}=\left(\begin{array}{rr}
-1 & 0 \\
0 & 1
\end{array}\right), \\
\tau_{3}=\tau_{7}=\tau_{3}^{-1}=\tau_{7}^{-1}=\left(\begin{array}{rr}
1 & 0 \\
0 & -1
\end{array}\right), \\
\tau_{4}=\tau_{8}=\tau_{4}^{-1}=\tau_{8}^{-1}=\left(\begin{array}{rr}
-1 & 0 \\
0 & -1
\end{array}\right) .
\end{gathered}
$$

For further system transformation (5) in accordance with the general ideas from works $[3,4]$, it is reasonable to use such denotations:

$$
\begin{aligned}
a & :=\frac{1}{\sqrt{\left(x-x_{0}\right)^{2}+\left(y-y_{0}\right)^{2}}}, \\
b & :=\frac{1}{\sqrt{\left(x+x_{0}\right)^{2}+\left(y-y_{0}\right)^{2}}}, \\
c & :=\frac{1}{\sqrt{\left(x-x_{0}\right)^{2}+\left(y+y_{0}\right)^{2}}}, \\
d & :=\frac{1}{\sqrt{\left(x+x_{0}\right)^{2}+\left(y+y_{0}\right)^{2}}},
\end{aligned}
$$




$$
\begin{aligned}
& e:=\frac{1}{\sqrt{\left(x-x_{0}\right)^{2}+\left(y-y_{0}\right)^{2}+4 h^{2}}}, \\
& f:=\frac{1}{\sqrt{\left(x+x_{0}\right)^{2}+\left(y-y_{0}\right)^{2}+4 h^{2}}}, \\
& g:=\frac{1}{\sqrt{\left(x-x_{0}\right)^{2}+\left(y+y_{0}\right)^{2}+4 h^{2}}}, \\
& h:=\frac{1}{\sqrt{\left(x+x_{0}\right)^{2}+\left(y+y_{0}\right)^{2}+4 h^{2}}} .
\end{aligned}
$$
basis:

Then, in the IE system (5) we will go to the new

$$
\begin{aligned}
\rho_{i}^{\prime}(x, y) & :=\rho_{i}\left[\tau_{i}^{-1} \cdot\left(\begin{array}{l}
x \\
y
\end{array}\right)\right], i=\overline{1,8} \\
f_{k}^{\prime}\left(x_{0}, y_{0}\right) & :=f_{k}\left[\tau_{k}^{-1} \cdot\left(\begin{array}{l}
x_{0} \\
y_{0}
\end{array}\right)\right], k=\overline{1,8} .
\end{aligned}
$$

On the basis of the implemented "change of variable", which is also used in the kernels of IE, the system (5) can be shown as

$$
\begin{aligned}
& \iint_{\Delta_{1}} \sum_{j=1}^{8} a_{i j}^{\prime} \rho_{j}^{\prime}(x, y) \mathrm{d} x \mathrm{~d} y=f_{i}^{\prime}\left(x_{0}, y_{0}\right), \\
& \left(x_{0}, y_{0}\right) \in(0, a) \times(0, b), \quad i=\overline{1,8},
\end{aligned}
$$

where

$$
A^{\prime}:=\left(a_{i j}^{\prime}\right)_{i, j=1}^{8}=\left\|\begin{array}{llllllll}
a & b & c & d & e & f & g & h \\
b & a & d & c & f & e & h & g \\
c & d & a & b & g & h & e & f \\
d & c & b & a & h & g & f & e \\
e & f & g & h & a & b & c & d \\
f & e & h & g & b & a & d & c \\
g & h & e & f & c & d & a & b \\
h & g & f & e & d & c & b & a
\end{array}\right\| .
$$

Thus, on this stage we have received the system of eight IEs where integration is performed only on one component surface $-S_{1}$.

In its turn, as sub-groups $\left\{e, \tau_{x}\right\},\left\{e, \tau_{y}\right\}$, $\left\{e, \tau_{z}\right\}$ are cyclic groups, thus, the table of their characters looks the same [5]:

\begin{tabular}{c|cc} 
& $e$ & $\tau$ \\
\hline$\chi^{1}$ & 1 & 1 \\
$\chi^{2}$ & 1 & -1
\end{tabular}

where $\tau:=\tau_{x} \vee \tau_{y} \vee \tau_{z}$. Thus, we can calculate the group characters of the eighth order $\left\{\tau_{i}\right\}_{i=1}^{8}$, taking direct product of matrices of characters (Fourier's matrices) of sub-groups $\left\{e, \tau_{x}\right\},\left\{e, \tau_{y}\right\},\left\{e, \tau_{z}\right\}$. As a result we will have

$$
F:=\left(F_{j i}\right)_{j, i=1}^{8}=\left(\begin{array}{rrrrrrrr}
1 & 1 & 1 & 1 & 1 & 1 & 1 & 1 \\
1 & -1 & -1 & 1 & 1 & -1 & -1 & 1 \\
1 & 1 & -1 & 1 & -1 & 1 & -1 & -1 \\
1 & -1 & 1 & 1 & -1 & -1 & 1 & -1 \\
1 & 1 & 1 & -1 & 1 & -1 & -1 & -1 \\
1 & -1 & -1 & -1 & 1 & 1 & 1 & -1 \\
1 & 1 & -1 & -1 & -1 & -1 & 1 & 1 \\
1 & -1 & 1 & -1 & -1 & 1 & -1 & 1
\end{array}\right) \text {. }
$$

After using forward and backward transformation of Fourier, the matrix $A^{\prime}$ can be reduced to the diagonal representation [4], and the system (6) can be "split" on eight independent IEs

$$
\begin{aligned}
& \iint_{\Delta_{1}} A_{j} \bar{\rho}_{j}(x, y) \mathrm{d} x \mathrm{~d} y=\bar{f}_{j}\left(x_{0}, y_{0}\right), \\
& \left(x_{0}, y_{0}\right) \in(0, a) \times(0, b), \quad j=\overline{1,8},
\end{aligned}
$$

where

$$
\bar{\rho}_{j}(x, y)=\sum_{i=1}^{8} F_{j i} \cdot \rho_{i}^{\prime}(x, y),(x, y) \in \Delta_{1},
$$

$\bar{f}_{j}\left(x_{0}, y_{0}\right)=\sum_{i=1}^{8} F_{j i} \cdot f_{i}^{\prime}\left(x_{0}, y_{0}\right)$, and $A_{j}-$ the elements of the diagonal matrix $F \cdot A^{\prime} \cdot F^{-1}$, which are calculated after the formulas:

$$
\begin{aligned}
& A_{1}=a+b+c+d+e+f+g+h, \\
& A_{2}=a-b-c+d+e-f-g+h, \\
& A_{3}=a+b-c+d-e+f-g-h, \\
& A_{4}=a-b+c+d-e-f+g-h, \\
& A_{5}=a+b+c-d+e-f-g-h, \\
& A_{6}=a-b-c-d+e+f+g-h, \\
& A_{7}=a+b-c-d-e-f+g+h, \\
& A_{8}=a-b+c-d-e+f-g+h .
\end{aligned}
$$

After solving approximately eight independent IEs (7) by the collocation method, and then the system (8), we will obtain the value of the functions $\rho_{i}^{\prime}(x, y) i=\overline{1,8}$, by the help of which it is possible to calculate the potential in any point of the space $(\bar{x}, \bar{y}, \bar{z})$ using the following formula:

$$
\begin{gathered}
u(\bar{x}, \bar{y}, \bar{z})=\iint_{\Delta_{1}}\left[\frac{\rho_{1}^{\prime}(x, y)}{\sqrt{\left(x_{1}^{\prime}-\bar{x}\right)^{2}+\left(y_{1}^{\prime}-\bar{y}\right)^{2}+(\bar{z}-h)^{2}}}+\right. \\
+\frac{\rho_{2}^{\prime}(x, y)}{\sqrt{\left(x_{2}^{\prime}+\bar{x}\right)^{2}+\left(y_{2}^{\prime}-\bar{y}\right)^{2}+(\bar{z}-h)^{2}}}+
\end{gathered}
$$




$$
\begin{gathered}
+\frac{\rho_{3}^{\prime}(x, y)}{\sqrt{\left(x_{3}^{\prime}-\bar{x}\right)^{2}+\left(y_{3}^{\prime}+\bar{y}\right)^{2}+(\bar{z}-h)^{2}}}+ \\
+\frac{\rho_{4}^{\prime}(x, y)}{\sqrt{\left(x_{4}^{\prime}+\bar{x}\right)^{2}+\left(y_{4}^{\prime}+\bar{y}\right)^{2}+(\bar{z}-h)^{2}}}+ \\
+\frac{\rho_{5}^{\prime}(x, y)}{\sqrt{\left(x_{5}^{\prime}-\bar{x}\right)^{2}+\left(y_{5}^{\prime}-\bar{y}\right)^{2}+(\bar{z}+h)^{2}}}+ \\
+\frac{\rho_{6}^{\prime}(x, y)}{\sqrt{\left(x_{6}^{\prime}+\bar{x}\right)^{2}+\left(y_{6}^{\prime}-\bar{y}\right)^{2}+(\bar{z}+h)^{2}}}+ \\
+\frac{\rho_{7}^{\prime}(x, y)}{\sqrt{\left(x_{7}^{\prime}-\bar{x}\right)^{2}+\left(y_{7}^{\prime}+\bar{y}\right)^{2}+(\bar{z}+h)^{2}}}+ \\
\text { where }\left(\begin{array}{l}
x_{i}^{\prime} \\
y_{i}^{\prime}
\end{array}\right)=\rho_{8}^{\prime}(x, y) \\
\mathrm{x} \bar{\tau}_{i}^{-1}\left(\begin{array}{l}
x \\
y
\end{array}\right), i=\overline{1,8} \mathrm{~d} y,
\end{gathered}
$$

The use of the approach offered above allows to test an initial task for the arbitrary boundary values of the potential. In the Pic. $2-3$ the distributing of lines of even potential is presented in the plane $x=0$ at different relations of the length (a) and width (b) of the plate, with the use of piecewise permanent approximation of IE density with the proper boundary values of the potential: $f_{1}=1$; $f_{2}=-100$. A number of collocation points at the separation of constituent $S_{1}$ equals 100 .

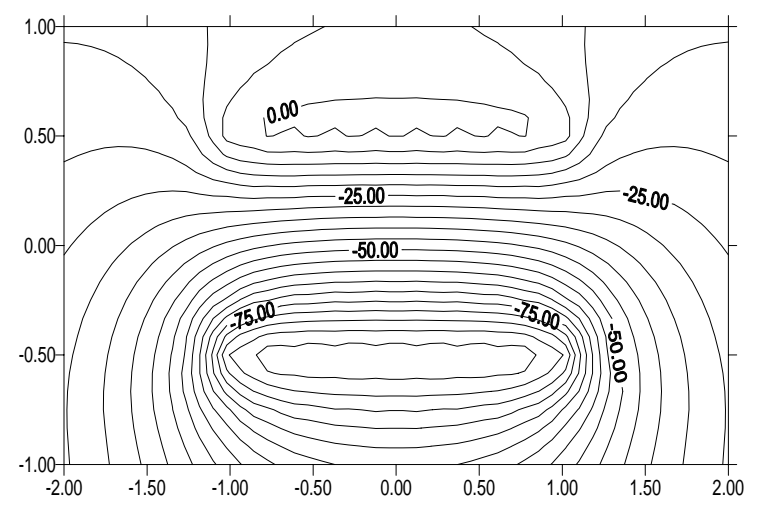

Pic. 2 - Distributing of level lines in the plane

$$
x=0 \text { at } a / b=1
$$

According to [6], for showing of the qualitative picture of the field in the plane, which forms as a result of central cross-section of the investigated system of the charged electrodes it is appropriate to use some "plane" approximation when solving a spatial problem. The Pic. 4 shows the distribution of lines of even potential when solving the proper "plane" task, and the Table 1 shows the value of the potential in some checkpoints when solving a spatial problem and proper "plane" approximation.

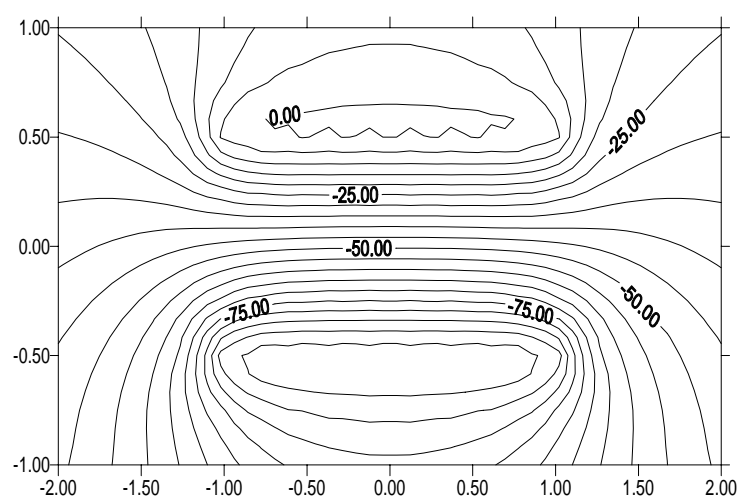

Pic. 3 - Distributing of level lines in the plane $x=0$ at $a / b=16$

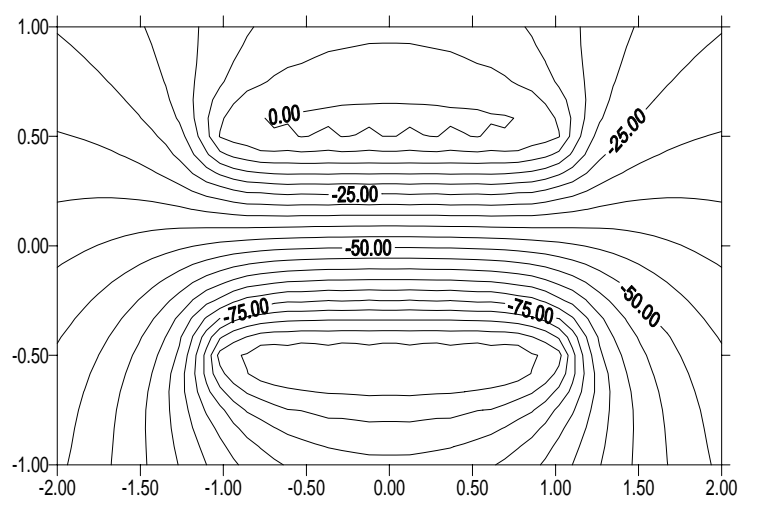

Pic. 4 - Distributing of level lines of “plane” task

Table 1. Potential of the electrostatic field in the points of plane $x=0$

\begin{tabular}{|c|c|c|}
\hline$(y, z)$ & $u(a / b=16)$ & $\begin{array}{c}\text { "plane" } \\
\text { approximation }\end{array}$ \\
\hline$(-1.00,-0.75)$ & -79.3731 & -79.3729 \\
\hline$(-1.00,-0.25)$ & -66.4593 & -66.4594 \\
\hline$(-1.00,0.00)$ & -46.3909 & -46.3910 \\
\hline$(-1.00,0.25)$ & -27.0001 & -27.0004 \\
\hline$(-1.00,0.75)$ & -10.4086 & -10.4082 \\
\hline$(-0.75,-0.50)$ & -99.9925 & -99.9930 \\
\hline$(1.00,0.75)$ & -10.4086 & -10.4082 \\
\hline$(2.00,-1.00)$ & -48.9304 & -48.9307 \\
\hline
\end{tabular}

It is necessary to notice that solving a spatial problem with the arbitrary boundary values of potentials on electrodes we should calculate a special additive constant $C$ which appears in the integral presentation of the field [6]. In our case $C=-49.5$. 


\section{A POSTERIORI METHOD OF ERROR EVALUATION}

Without diminishing general attitude, we will consider one of the eight independent IEs (7):

$$
\begin{aligned}
& \left(K \bar{\rho}_{1}\right)\left(x_{0}, y_{0}\right) \equiv \iint_{\Delta_{1}} A_{1} \bar{\rho}_{1}(x, y) \mathrm{d} x \mathrm{~d} y= \\
& =\bar{f}_{1}\left(x_{0}, y_{0}\right), \quad\left(x_{0}, y_{0}\right) \in(0, a) \times(0, b) .
\end{aligned}
$$

An approximate solution to this equation is found by the collocation method with the use of piecewise permanent basic functions. Element division is conducted according to $\Delta_{1}=[0, a] \times[0, b]$. By "Extremal" is considered an element $D_{N_{x} N_{y}}:=\left[a-h_{x}, a\right] \times\left\lfloor b-h_{y}, b\right\rfloor$, which corresponds the angular point of constituent $S_{1}$ (see. Pic. 5). Here $h_{x}=\frac{a}{N_{x}}, h_{x}=\frac{b}{N_{x}}$, where $N_{x}, N_{y}$ - the number of points of segment division $[0, a],[0, b]$, accordingly. It is necessary to notice that without accounting the present symmetry in geometry of the open surfaces we should have taken into account the peculiarities of solving in the circuit of eight angular points of surface $S$, which substantially complicates all algorithm of calculations.

It is known [7] that the "density of charge distribution" $\bar{\rho}_{1}(x, y)$ in the circuit of the "angular point" $Q:=(a, b)$ and reaching the circuit has a feature which is expressed by the formula

$$
\Omega(x, y)=\frac{[(a-x) \cdot(b-y)]^{1 / 2}}{(a-x)^{\gamma}+(b-y)^{\gamma}},
$$

where $\gamma \approx 1,7034$. To remove this peculiarity it is necessary to introduce a special change of variables in the proper double integrals. However, it considerably complicates the algorithm of approximate solving of the integral equation and does not enable to use a simple and effective numeral and analytical scheme [8]. Therefore, we will take into account this peculiarity by net condensing in the circuit of the point $Q$ to achieve the accuracy of calculations and we will use general ideas mentioned in the works $[9,10]$.

Let us suppose that in the result of solving the systems of linear algebraic equations, which approximates the proper operator equation, we get an approximate solution $\bar{\rho}_{1 \varepsilon}(P)$, the analysis of which in any case requires the investigation of properties of its error $\varepsilon_{\rho_{1}}:=\bar{\rho}_{1}-\bar{\rho}_{1 \varepsilon}$.

Let us put some limits and create approximation $e_{\rho_{1}}$ to the real error $\varepsilon_{\rho_{1}}$. The verification of satisfying boundary condition consists in calculating the potential in some checkpoint $P_{1}$, which lies in the circuit of the angular point of the constituent $S_{1}$ and corresponds to the "extremal" element $D_{N_{x} N_{y}}$. Then we will give the error function $e_{\rho_{1}}$ as $e_{\rho_{1}}=\lambda_{1} \cdot B_{P_{1}}(x, y)$ and we will find an unknown parameter $\lambda_{1}$ by collocation (9) exactly in the point

$$
\begin{aligned}
& P_{1}:=\left\{a-\frac{h_{x}}{4}, b-\frac{h_{y}}{4}\right\}: \\
& \lambda_{1}=\frac{\bar{f}_{1}\left(P_{1}\right)-\left(K \bar{\rho}_{1}\right)\left(P_{1}\right)}{\left(K B_{P_{1}}\right)\left(P_{1}\right)} .
\end{aligned}
$$

It should be noticed that such simple formula for parameter $\lambda_{1}$ calculating is connected with finiteness of the bilinear bubble-function $B_{P_{1}}(x, y)$ :

$$
\begin{gathered}
\quad \operatorname{supp} B_{P_{1}}(x, y)=D_{N_{x} N_{y}}^{1 / 4}:= \\
=\left[a-\frac{h_{x}}{2}, a\right] \times\left[b-\frac{h_{y}}{2}, b\right] .
\end{gathered}
$$

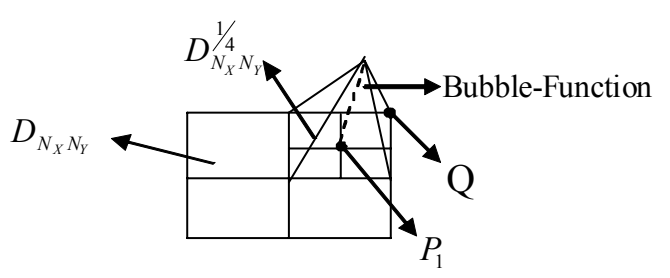

Pic. 5 - Schematic presentation of the "extremal" element

Calculating the denominator in the formula (10) it is convenient to take an element $D_{N_{x} N_{y}}^{1 / 4}$ to the local coordinate system $(\alpha, \beta)$ so that $|\alpha| \leq 1$, $|\beta| \leq 1$. It enables the presentation of $\left(K B_{P_{1}}\right)\left(P_{1}\right)$ as a sum of four integrals $I_{1}, I_{2}, I_{3}, I_{4}$ :

$$
\mathrm{I}_{1}:=\int_{x_{i}-\widetilde{\Delta}_{1}}^{x_{i}} \int_{y_{j}-\widetilde{\Delta}_{2}}^{y_{j}} A_{1} \cdot B_{P_{1}}^{1}(x, y) \mathrm{d} x \mathrm{~d} y,
$$




$$
\begin{aligned}
& \mathrm{I}_{2}:=\int_{x_{i}}^{x_{i}+\widetilde{\Delta}_{1}} \int_{y_{j}-\widetilde{\Delta}_{2}}^{y_{j}} A_{1} \cdot B_{P_{1}}^{2}(x, y) \mathrm{d} x \mathrm{~d} y, \\
& \mathrm{I}_{3}:=\int_{x_{i}}^{x_{i}+\widetilde{\Delta}_{1}} \int_{y_{j}+\widetilde{\Delta}_{2}}^{y_{j}} A_{1} \cdot B_{P_{1}}^{3}(x, y) \mathrm{d} x \mathrm{~d} y, \\
& \mathrm{I}_{4}:=\int_{x_{i}-\widetilde{\Delta}_{1}}^{x_{i}} \int_{y_{j}}^{y_{j}+\widetilde{\Delta}_{2}} A_{1} \cdot B_{P_{1}}(x, y) \mathrm{d} x \mathrm{~d} y,
\end{aligned}
$$

where $\widetilde{\Delta}_{1}=\frac{\mathrm{h}_{\mathrm{x}}}{4}, \widetilde{\Delta}_{2}=\frac{\mathrm{h}_{\mathrm{y}}}{4}$, in each of them to perform some change of variables. So, for example, for $I_{1}$ we will have

$$
\left\{\begin{array}{l}
x(\alpha, \beta):=\frac{2 x_{i}-\widetilde{\Delta}_{1}}{2}+\frac{\widetilde{\Delta}_{1}}{2} \cdot \alpha, \\
y(\alpha, \beta):=\frac{2 y_{j}-\widetilde{\Delta}_{2}}{2}+\frac{\widetilde{\Delta}_{2}}{2} \cdot \beta,-1 \leq \alpha, \beta \leq 1 .
\end{array}\right.
$$

Thus, the proper constituents of the bubble-function in the local coordinates are as following

$$
\begin{aligned}
& B^{1}(\alpha, \beta)=\frac{1}{4}(1+\alpha)(1+\beta), \\
& B^{2}(\alpha, \beta)=\frac{1}{4}(1-\alpha)(1+\beta), \\
& B^{3}(\alpha, \beta)=\frac{1}{4}(1-\alpha)(1-\beta), \\
& B^{4}(\alpha, \beta)=\frac{1}{4}(1+\alpha)(1-\beta) .
\end{aligned}
$$

We use the above mentioned values for a posteriori error evaluation on the "extremal" element to find the approximate solution (9) with the beforehand accuracy. As a criterion of stopping the clarification process of the approximate solution $\bar{\rho}_{1 \varepsilon}(P)$ there is some indicator

$$
\eta_{\rho_{1}}:=\frac{\left\|e_{\rho_{1}}(x, y)\right\|_{L_{2}}}{\sqrt{\left\|e_{\rho_{1}}(x, y)\right\|_{L_{2}}^{2}+\left\|\bar{\rho}_{1 \varepsilon}(x, y)\right\|_{L_{2}}^{2}}} \cdot 100 \% \text {. }
$$

If the indicator (11) does not exceed set possible level, then we stop the clarification $\bar{\rho}_{1 \varepsilon}$, otherwise we carry out the net condensing. In our case, on the first step of net condensing we will get four new elements $\left(D_{N_{x} N_{y}}^{1 / 4}\right)_{i},(i=\overline{1,4})$.

The Table 2 shows the value of the potential in some checkpoints which are situated in the circuit of the "extremal" element before $\left(u_{0}\right)$ and after $\left(u_{1}\right)$ using a posteriori error appraiser at a possible level of $10 \%$.
Table 2. Potential at some checkpoints in the case of piecewise-bilinear bubble function

\begin{tabular}{|c|c|c|c|c|}
\hline \multirow{2}{*}{$N_{y}$} & $y$ & 0,9375 & 0,8750 & 0,8125 \\
\cline { 3 - 5 } & $z$ & 0,9375 & 0,9375 & 0,9375 \\
\hline \multirow{4}{*}{4} & $u_{0}$ & 0,8963 & 0,9454 & 0,9697 \\
& $u_{1}$ & 1,0358 & 1,0189 & 1,0105 \\
& $\pi_{1}$ & 0,1395 & 0,0735 & 0,0408 \\
& 3 & 0,2116 & 0,1132 & 0,0631 \\
\hline \multirow{5}{*}{5} & $u_{0}$ & 0,9321 & 0,9792 & - \\
& $u_{1}$ & 1,0234 & 1,0072 & - \\
& $\pi_{1}$ & 0,0913 & 0,0280 & - \\
& 3 & 0,1365 & 0,0421 & - \\
\hline \multirow{5}{*}{6} & $u_{0}$ & 0,9600 & - & - \\
& $u_{1}$ & 1,0138 & - & - \\
& $\pi_{1}$ & 0,0538 & - & - \\
& 3 & 0,0794 & - & - \\
\hline
\end{tabular}

\section{ITERATION REFINEMENT OF SOLUTIONS}

Using the method of a posteriori evaluation of error partly offered above, we will consider the iteration process of clarification of the approximate solutions to the problem (9). On the basis of preliminary received densities using the collocation method $\bar{\rho}_{j}(x, y) j=\overline{1,8} \quad 3 \quad$ (7) in the points $\left(x_{i}, y_{p}\right)$, where

$$
\begin{gathered}
x_{i}=(2 i-1) \cdot \frac{h_{x}}{2}, i=\overline{1, N_{x}}, \\
y_{p}=(2 p-1) \cdot \frac{h_{y}}{2}, p=\overline{1, N_{y}},
\end{gathered}
$$

we find the value $\bar{\rho}_{j}(x, y)$ in the mesh nodes $\left(\tilde{x}_{k}, \tilde{y}_{l}\right)$, where $\tilde{x}_{k}=(k-1) \cdot h_{x}, k=\overline{1, N_{x}+1}$, $\tilde{y}_{l}=(l-1) \cdot h_{y}, l=\overline{1, N_{y}+1}$, as follows:

1) in the extreme points of the net, for example, in the point $\left(\tilde{x}_{1}, \tilde{y}_{N_{y}+1}\right), \bar{\rho}_{j}\left(\tilde{x}_{1}, \tilde{y}_{N_{y}+1}\right):=\bar{\rho}_{j}\left(x_{1}, y_{N_{y}}\right)$;

2) for the points $\left(\widetilde{x}_{k}, \widetilde{y}_{N_{y}+1}\right), k=\overline{1, N_{x}}$,

$$
\bar{\rho}_{j}\left(\tilde{x}_{k}, \tilde{y}_{N_{y}+1}\right):=\frac{1}{2}\left[\bar{\rho}_{j}\left(x_{i}, y_{N_{y}}\right)+\bar{\rho}_{j}\left(x_{i+1}, y_{N_{y}}\right)\right],
$$

where $i=\overline{1, N_{x}-1}$;

3) in the points $\left(\tilde{x}_{k}, \tilde{y}_{l}\right), k=\overline{2, N_{x}}, l=\overline{2, N_{y}}$, we perform

$$
\begin{gathered}
\bar{\rho}_{j}\left(\tilde{x}_{k}, \tilde{y}_{l}\right):=\frac{1}{4}\left[\bar{\rho}_{j}\left(x_{i}, y_{p}\right)+\bar{\rho}_{j}\left(x_{i+1}, y_{p}\right)+\right. \\
+\bar{\rho}_{j}\left(x_{i}, y_{p+1}\right)+\bar{\rho}_{j}\left(x_{i+1}, y_{p+1}\right),
\end{gathered}
$$

where $i=\overline{1, N_{x}-1}, p=\overline{1, N_{y}-1}$.

Then, we calculate the potential in the mesh 
nodes $\left(\tilde{x}_{k}, \tilde{y}_{l}\right)$. On the basis of the bubble-function, by analogy with (10), we receive $\tilde{\lambda}_{j}$. On the basis of the discovered values of density in the mesh nodes we re-count $\bar{\rho}_{j}(x, y)$ in the points $\left(x_{i}, y_{p}\right)$ after the rules described above 1)-3). Then the procedure of iteration clarification can repeat. The criterion of stopping of such calculations can be, for example, fixing of a number of iterations or under the condition of $\left\|e_{\rho_{j}}(x, y)\right\|_{L_{2}} \leq$ eps .

The Table 3 shows the potential value in some checkpoints before and after the use of iteration refinement of solutions.

Table 3. Potential $u$ at some checkpoints after the use of iteration refinement of solution $(\mathbf{e p s}=\mathbf{0 , 0 1})$

\begin{tabular}{|c|c|c|c|c|c|}
\hline \multirow{2}{*}{$y$} & \multirow{2}{*}{$z$} & \multicolumn{5}{|c|}{ Iteration } \\
\cline { 3 - 6 } & & 1 & 2 & 3 & 4 \\
\hline 0,95 & 0,95 & 0,9029 & 0,8150 & 0,9357 & 0,9851 \\
0,80 & 0,95 & 0,9650 & 0,8851 & 1,0318 & - \\
0,60 & 0,95 & 0,9545 & 0,8892 & 1,0689 & - \\
0,40 & 0,95 & 0,9569 & 0,8868 & 1,0786 & - \\
0,20 & 0,95 & 0,9579 & 0,8876 & 1,0851 & - \\
0,80 & 0,80 & 1,0338 & - & - & - \\
0,60 & 0,60 & 1,0000 & - & - & - \\
\hline
\end{tabular}

\section{CONCLUSIONS}

Taking an example of the numerical solving one spatial problem the problems at calculating of the electrostatic field are investigated. Approximate solutions are received on the basis of the IE method taking into account the present symmetry in geometry of separate elements of the surface. It allowed to carry on solving the eight independent IEs, where integration is conducted for $1 / 8$ of the whole surface. It, in its turn, enables to decrease random-access memory of the computer when forming the system of linear algebraic equations, which approximates the proper integral equation in 64 times and to avoid numerical instability which can arise at slight increase of the solved systems. This approach also enables instead of eight special points of the surface where it is necessary to take into account the singular way of the solution to control only one. In the circuit of this "extremal" element a feature is taken into account on the basis of the created a posteriori method of error evaluation. For clarification of the solutions in the mesh nodes the iteration process is introduced. All the advantages of this method were confirmed by numerical experiments.

\section{REFERENCES}

[1] Lions J.-L., Magenes E. Problŭmes aux Limites non Homogŭnes et Applications. Vol. 1. Dunod. Paris, 1968.

[2] Sybil Yu.M. Three dimensional elliptic boundary value problems for an open Lipschitz surface // Mathematical Studies. 1997. Vol. 8. № 2. P. 79-96.

[3] Zakharov Y.V., Safronov S.I., Tarasov R.P. The Method of numeral solving of integral equations in the boundary problems with the Abelian group of symmetries of the finite order // Journal of Computational Mathematics and Mathematical Physics. 1990. Vol. 30. № 11. P. 1661-1674 (in Russian).

[4] Zakharov Y.V., Safronov S.I., Tarasov R.P. The Abelian groups of the finite order in the numerical analysis of linear boundary problems of the potential theory // Journal of Computational Mathematics and Mathematical Physics. 1992. Vol. 32. № 1. P. 40-58 (in Russian).

[5] Serr G.P. Linear presentations of finite groups. M.: RKHD, 2003. 132 p. (in Russian).

[6] Harasym Y.S., Mochurad L.I., Ostudin B.A. Paralling of the method of integral equations solving the boundary problems of the potential theory of potential with the symmetric boundary elements // Materials of the Third International Scientific and Technical Conference "Computer Sciences and Information Technologies", September, 25-27 2008, Lviv: "Tower and Co". - 2008. - P. 342346 (in Ukrainian).

[7] Morrison J.A., Lewis J.A. Charge singularity at the corner of a fllat plate// SIAM J. Appl. Math. Vol. 31. № 2. 1976. P. 233-249.

[8] Garasym Ya.S., Ostudin B.A. On numerical appoach to solve some three-dimensional boundary value problems in potential theory based on integral equation method // Journal of Computational and Applied Mathematics. 2003. № 1 (88). P. 17-28.

[9] Eriksson K., Estep D., Hansbo P., Johnson C. Introduction to Adaptive Method for Differential Equation // Acta Numerica. - 1955. P. 1-54.

[10] Carstensen C., Maischak M., Praetorins D., Stephan E.P. Residual based a posteriori estimate for hypersingular equation on surfaces // Numer. Math. - 2004. - 97. P. 397425 . 
Lesya Mochurad was born in Novyy Rozdil, Lviv Region, Ukraine in April 14, 1984. In 2006 she graduated from Ivan Franko National University in Lviv, Faculty of Applied Mathematics and Computer Science (master's degree). Since 2006 she has been a post-graduate student at Ivan Franko National University in Lviv, Faculty of Applied Mathematics and Computer Science. Scientific interests: method of the numerical solving integral equations in the boundary problems of the potential theory with the Abelian group of symmetries of the finite order and decomposition of complicated areas.

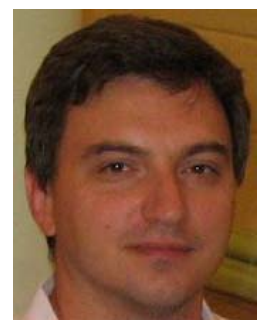

Yaroslav Harasym was born in Stavropol Territory, the Russian Federation, in March 29, 1969, Higher Education. Since 2008 he has been working as a senior teacher at the department of computational mathematics at Ivan Franko National University in Lviv. Scientific interests: numerical solving integral equations in the boundary problems in mathematical physics for open surfaces.

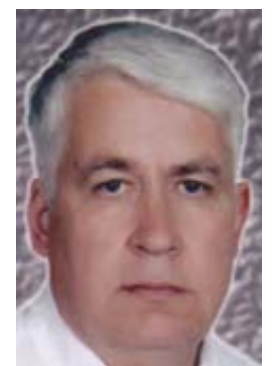

Borys Ostudin was born in Lviv May 3, 1947, Candidate of Physical and Mathematical Sciences degree. Since 1982 he has been working as an associate professor at the department of computational mathematics at Ivan Franko National University in Lviv. Scientific interests: numerical solving one- and two-dimensional integral first kind equations with weak singularities in kernels. 